\title{
A coherent optical link through the turbulent atmosphere
}

\author{
Khelifa Djerroud, ${ }_{1}^{1}$ Ouali Acef, ${ }_{1}^{1}$ André Clairon, ${ }^{1}$ Pierre Lemonde, ${ }^{1}$ \\ Catherine N. Man, ${ }^{2}$ Etienne Samain, ${ }^{3}$ and Peter Wolf ${ }^{1, *}$ \\ ${ }^{1}$ LNE-SYRTE, Observatoire de Paris, CNRS, UPMC, 61 av. de l'Observatoire, \\ 75014 Paris, France \\ ${ }^{2}$ ARTEMIS, Observatoire de la Côte d'Azur, Univ. Nice, CNRS, 06304 Nice Cedex, France \\ ${ }^{3}$ GéoAzur, Observatoire de la Côte d'Azur, Univ. Nice, CNRS, 06460 St Vallier de Thiey, \\ France \\ *Corresponding author: Peter.Wolf@obspm.fr
}

\begin{abstract}
We describe the realization of a $5 \mathrm{~km}$ free space coherent optical link through the turbulent atmosphere between a telescope and a ground target. We present the phase noise of the link, limited mainly by atmospheric turbulence and mechanical vibrations of the telescope and the target. We discuss the implications of our results for applications, with particular emphasis on optical Doppler ranging to satellites and long distance frequency transfer. (c) 2018 Optical Society of America

OCIS codes: 010.1330, 120.5050, 060.2605.
\end{abstract}

Atomic clocks have been improving rapidly over the past years and are now reaching uncertainties in relative frequency of 2 parts in $10^{17}$ after less than $5 \times 10^{4}$ seconds of integration time [1]. Applications of such clocks in fundamental physics, geodesy, navigation etc... require their comparison over large distances without degrading their performance. In the longer term one expects applications of such clocks on board terrestrial and solar system satellites [2,3], which require a high performance free space link from ground to space and/or between spacecraft. At present, no existing long distance comparison method reaches the required level of uncertainty [4], and even the improved microwave link of the ACES (Atomic Clock Ensemble in Space) mission [5] or the optical T2L2 (Time Transfer by Laser Link) link [6] will require several days of integration time to reach $10^{-17}$. Over short to medium distances $\left(\approx 10^{2} \mathrm{~km}\right)$ optical fibre links have demonstrated sufficient performance [7], and our aim is to extend those methods to free space propagation and towards ground to 
satellite and intercontinental (via a relay satellite) frequency comparison of clocks. Other applications of coherent free space links are optical satellite Doppler ranging and broadband optical communications, the latter being the focus of much attention in recent years with the promise of $\mathrm{Gb} / \mathrm{s}$ data rates over large distances. At such high rates the main limitation is strong amplitude fluctuation (scintillation) due to atmospheric turbulence, which has been investigated experimentally in a $142 \mathrm{~km}$ coherent optical link between two Canary islands [8]. In contrast, we focus on the low frequency $(<1 \mathrm{kHz})$ part of the link phase noise spectrum, of relevance to clock comparisons and optical Doppler ranging, which is to a large extent independent of amplitude fluctuations. Recently a short baseline $(10 \mathrm{~m})$ laboratory experiment [9] and a roof-top experiment over a $100 \mathrm{~m}$ distance have been reported [10]. The latter concludes that free-space coherent optical links may only be suitable for short distance $(<1 \mathrm{~km})$ clock comparisons. We arrive at the opposite conclusion showing not only that such links display high performance over our $5 \mathrm{~km}$ distance, but also that they hold great promise for satellite to ground links, with the perspective of reaching the performance of the best optical clocks in less than $1000 \mathrm{~s}$ integration time. In this letter we present a brief description of the experiment and our main results, with more detailed information relegated to a forthcoming publication.

Our experiment took place at the Observatoire de la Côte d'Azur (OCA) lunar and satellite laser ranging facility located on the plateau de Calern at an altitude of $1323 \mathrm{~m}$, with data taken during several days in June and July 2009. The set up consists of a heterodyne Michelson interferometer with unequal arm lengths (see fig. 1), using a $1064 \mathrm{~nm} \mathrm{Nd:YAG}$ laser (Innolight/Prometheus, $\approx 1 \mathrm{kHz}$ linewidth). The local arm is frequency shifted by $200 \mathrm{MHz}$ using a Acousto-Optic Modulator (AOM) and recombined with the distant arm on the photodiode. The resulting heterodyne beat signal is used to phase lock a Voltage Controlled Oscillator (VCO) with the locking bandwidth set to around $50 \mathrm{kHz}$. The frequency of the VCO is counted using a zero dead-time counter with a $1 \mathrm{kHz}$ data rate. We also generate a quadrature signal (mixer M2) with respect to the phase locked loop (mixer M1), which is proportional to the beat signal amplitude but independent of its phase (to first order) when the loop is closed. It thus allows monitoring of the signal amplitude. The distant arm is fed into the Lunar Laser Ranging $1.5 \mathrm{~m}$ aperture telescope at OCA. The beam diameter at the exit of the telescope is about $380 \mathrm{~mm}$. The distant arm is reflected by a $5 \mathrm{~cm}$ corner cube mounted on an iron structure $\approx 3.5 \mathrm{~m}$ off the ground on a mountain top $\approx 2.5 \mathrm{~km}$ from the telescope. We feed $\approx 275 \mathrm{~mW}$ of optical power into the telescope for a received average power on the photodiode of $\approx 20 \mu \mathrm{W}$. The main sources of loss are the small size (relative to the beam diameter) of the corner cube and the low transmission of the telescope at $1064 \mathrm{~nm}$ (optimized for $532 \mathrm{~nm}$ ). As expected, we observe large amplitude fluctuations in the heterodyne beat signal, caused by scintillation from atmospheric turbulence. 


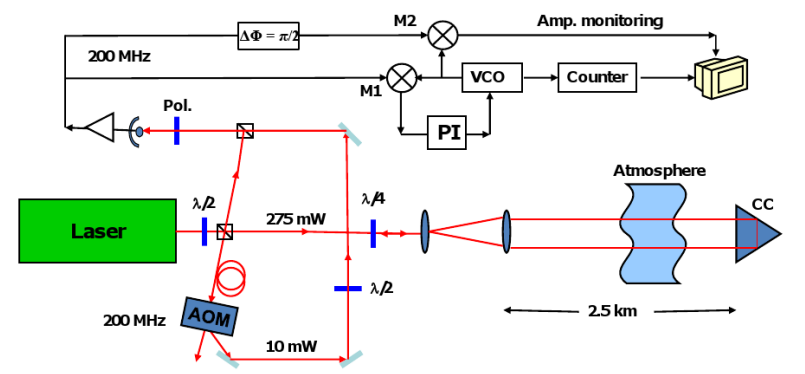

Fig. 1. Principle of the experiment: AOM, Acousto-Optic Modulator; Pol., Polarizer; PI, Proportional-Integrator filter; VCO, Voltage Controlled Oscillator.

In a ground-ground or ground-satellite free space coherent optical link the main limitation in the low frequency part of the spectrum is expected to arise from fluctuation of the refractive index of the atmosphere due to turbulence. Models to estimate the phase noise from turbulence can be found in [11] and references therein. Using those models for our horizontal $2.5 \mathrm{~km}$ return path we obtain a phase noise estimate about two orders of magnitude worse than for a zenithal ground to satellite link from the same location. Therefore, in order to obtain conditions representative of a vertical link, we have taken data during the calm periods of inversion of the temperature gradient (about $1 \mathrm{~h}$ after sunrise) where the observed turbulence level was significantly lower than during the rest of the day. This is also confirmed by the fact that the observed noise during those calm periods was close to the one reported in [12] obtained by interferometric observation of light from stars crossing the atmosphere vertically during average turbulence conditions. In [12] turbulence is quantified by the phase noise structure function defined as $D_{x}(\tau)=\left\langle[x(t+\tau)-x(t)]^{2}\right\rangle$ where $x(t)$ is the optical path length traveled by the signal received at time $t$. The obtained structure functions for $0.1 \mathrm{~s} \leq \tau \leq 10 \mathrm{~s}$ can be described by a power law of the form $D_{x}(\tau) \simeq C \tau^{\beta}$, with typical values in [12] of $D_{x}(1 \mathrm{~s}) \approx 20 \mu \mathrm{m}^{2}$ and $\beta \approx 1.45$ and large variations around those values. We typically obtain structure functions following a similar power law with $D_{x}(1 \mathrm{~s}) \approx 25 \mu \mathrm{m}^{2}$ and $\beta \approx 1.25$, which compare well with the results of [12]. Both results show significantly lower power law slopes than predicted from standard Kolmogorov turbulence theory $(\beta=5 / 3)$. We thus estimate that the phase noise from turbulence on our horizontal link during calm periods is representative (at least in order of magnitude) of the phase noise expected in a one-way ground-satellite link in average conditions.

Figure 2 shows the power spectral density (PSD) of fractional frequency fluctuations for a 33 min data set taken on July $3(\approx 1 \mathrm{~h}$ after sunrise) in clear sunny conditions. Similar results are obtained for other days in similar conditions. The lower curve represents our noise 


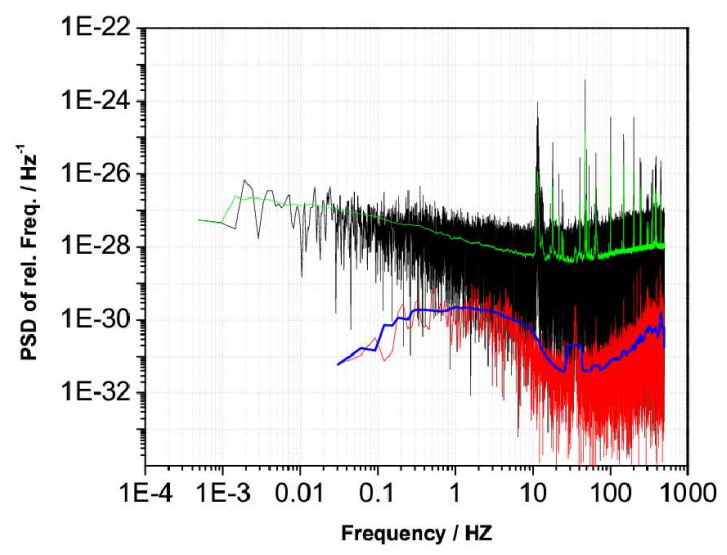

Fig. 2. Fractional frequency power spectral density of the free-space link (black) and system floor (red), with 500 points moving averages superposed in green and blue.

floor, obtained by placing a mirror on the distant arm before injection into the telescope. It is dominated at high Fourier frequency by the white phase noise of our counter, and below $30 \mathrm{~Hz}$ by acoustic noise in the laboratory. The upper curve is our measurement over the $5 \mathrm{~km}$ return distance through the turbulent atmosphere. The initial data consists of about $2 \times 10^{6}$ frequency measurements at $1 \mathrm{~ms}$ intervals, with a standard deviation of $96 \mathrm{~Hz}$. Optical cycle slips of the phase-locked loop are easily identified, as at our $1 \mathrm{~ms}$ sampling they correspond to $1 \mathrm{kHz}$ steps. We removed 66 such points $\left(\approx 3 \times 10^{-5}\right.$ of the data) all of which were obvious cycle slips and correlated well with measured signal extinction due to atmospheric scintillation. The PSD of our measurement shows turbulence noise at frequencies below $10 \mathrm{~Hz}$. For this part the PSD is proportional to $f^{-0.3}$, below the expected value from turbulence theory of $f^{-2 / 3}$ as already mentioned above. Above $10 \mathrm{~Hz}$ the PSD exhibits peaks due to mechanical resonances of the telescope and the target with amplitudes around $1 \mu \mathrm{m}$, but we also note an underlying increase of the PSD with frequency, due to amplitude to phase noise conversion in our phase locked loop.

Figure 3 shows the Allan deviation of fractional frequency $\left(\sigma_{y}(\tau)\right)$ of the same data set. At $\tau<0.1 \mathrm{~s}$ it is dominated by the sum of the periodic effects due to mechanical vibrations, and at larger $\tau$ its slope reflects the noise from atmospheric turbulence $\left(\propto \tau^{-0.3}\right)$, somewhat different from the expected value from turbulence theory $\left(\propto \tau^{-1 / 6}\right)$, as already mentioned above and consistent with measurements of [12].

The Allan deviation of our data is about $1.3 \times 10^{-14}$ at $\tau=1 \mathrm{~s}$, and reaches $2 \times 10^{-15}$ after about $100 \mathrm{~s}$ integration time, which is a remarkable stability for an uncompensated 


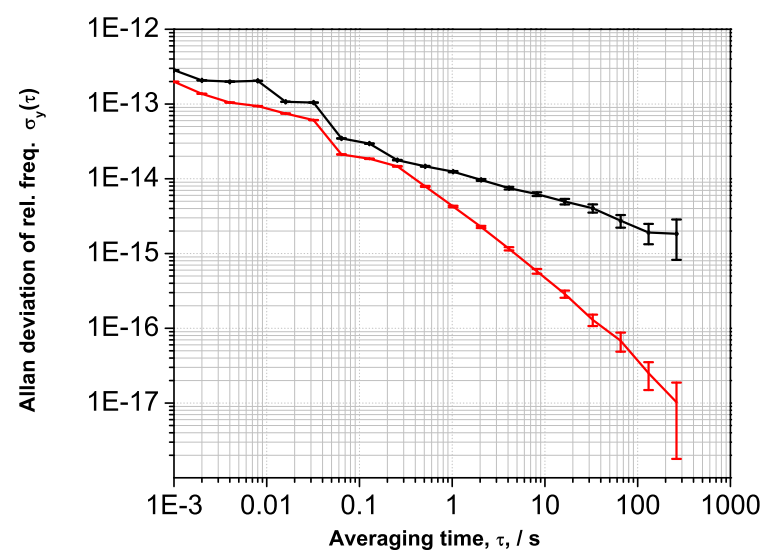

Fig. 3. Fractional frequency stability measured on the ground-ground free space link (black), and estimated for a ground to geostationary satellite clock comparison (red).

link through the turbulent atmosphere. For instance, it is similar to the uncompensated noise in the best $10^{2} \mathrm{~km}$ fibre links [7]. Given that, with appropriate two-way compensation schemes, those links show the by far best performance for frequency comparisons over short to medium distances, we believe that if our results are representative of a ground-satellite link, free space coherent optical links have excellent potential for future ground to space and intercontinental clock comparisons. This is discussed more quantitatively below.

Let us assume a two-way free space optical link between a ground station and a geostationary satellite, with heterodyne frequency measurements of the received signal vs. the local signal, on board $\left(y_{s}\left(t_{s}\right)\right)$, and on the ground $\left(y_{g}\left(t_{g}\right)\right)$. The up and down links will be affected by three main noise sources: satellite motion, ground station motion, and atmospheric turbulence. We further assume that the latter two are characterized by our measured noise as shown above. The two way system is used in such a way that the "up" signal is received at the satellite at the time of emission of the "down" signal (to within a few ms), which can easily be implemented during the data analysis when combining the on board and ground measurements. The frequency difference of the ground and on board optical clocks is given by $\Delta y=\left(y_{s}\left(t_{s}\right)-y_{g}\left(t_{g}\right)\right) / 2$. In that difference the Doppler effect from satellite motion cancels to a large extent (exactly if the two signals are coincident at the satellite). However, the noise from ground station motion and atmospheric turbulence partially remains as the emission of the "up" signal is separated from the reception of the "down" signal by the return travel time $\Delta t \simeq 250 \mathrm{~ms}$. We can estimate that residual noise $\left(y_{\text {res }}(t)\right)$, by calculating 
$y_{\text {res }}(t)=(y(t)-y(t+\Delta t)) / 2$ from our data, and investigate its statistics. The resulting PSD is given by the one in fig. 2 multiplied by a transfer function equal to $(1-\cos (2 \pi f \Delta t)) / 2$, resulting in a steep decrease at low frequency $(\leq 1 \mathrm{~Hz})$. The corresponding Allan deviation of $y_{\text {res }}(t)$ is shown in fig. 3. It stays a factor $\simeq \sqrt{2}$ (due to the factor 2 in $y_{\text {res }}$ ) below the measured noise at small $\tau$, but shows a steep decrease starting at $\tau \approx 250 \mathrm{~ms}$, reaching $1 \times 10^{-17}$ after about $300 \mathrm{~s}$ integration time. Thus, free space optical links hold great promise for future long distance clock comparisons, with the potential of reaching the uncertainty of the best present day optical clocks in less than $1000 \mathrm{~s}$.

A limit to the above argument is imposed by signal outages due to atmospheric scintillation and corresponding possible optical cycle slips. These will be uncorrelated between the up and down links, and will therefore fully affect $y_{\text {res }}(t)$. In our 33 min data set we observed 66 such signal outages, which implies an average loss of about 0.05 cycles per second. The resulting noise corresponds to an Allan variance of $\sigma_{y}(\tau) \approx 2 \times 10^{-16} / \sqrt{\tau}$ still significantly better than the best present optical clocks and just below the estimated stability shown in Fig. 3. If these cycle slips are not random they cause a frequency bias at worst equal to $\approx 2 \times 10^{-16}$. This does not affect the clock comparison as long as turbulence is sufficiently low to allow identification and removal of cycle slips (clearly the case in our data, see discussion above), and we expect this to be the case at most astronomical observing sites. Furthermore, signal outages can be mitigated by the use of adaptive optics schemes.

For Doppler ranging the residual noise on the satellite Doppler (and thus velocity) is given by $y_{\text {res }}(t)=(y(t)+y(t+\Delta t)) / 2$. The Allan deviation of the corresponding residual distance noise is $\sigma_{x}(\tau)=28 \mathrm{~nm}$ at $\tau=1 \mathrm{~ms}$ and $\sigma_{x}(\tau)=1.4 \mu \mathrm{m}$ at $\tau=1 \mathrm{~s}$. Although this corresponds to $>3$ orders of magnitude improvement on the present measurement noise in satellite laser ranging, one should bear in mind that, for lower orbits, the long term noise will be dominated by changes of the tropospheric delay as the satellite passes overhead (varying elevation), which can presently only be modeled at the mm level at best.

In conclusion, we have demonstrated operation of a free space coherent optical link through the turbulent atmosphere. Based on our results we estimate that such links promise large improvements in long distance clock comparisons and satellite ranging. We are at present working towards extending our experiment to a ground-space link using existing low orbit satellites equipped with corner cube reflectors. The main challenges in doing so are the large induced Doppler shift of our signal $( \pm 12 \mathrm{GHz})$, the low expected return power $<1 \mathrm{pW}$, and the longer signal travel time which requires frequency stabilization of our laser.

Acknowledgements: Valuable help by D. Albanese, S. Bize, M. Lours, J. Paris, J. Pinto, G. Santarelli, J-M. Torre is gratefully acknowledged. 


\section{References}

[1] Rosenband, T.; Hume, D. B.; Schmidt, P. O.; Chou, C. W.; Brusch, A.; Lorini, L.; Oskay, W. H.; Drullinger, R. E.; Fortier, T. M.; Stalnaker, J. E.; Diddams, S. A.; Swann, W. C.; Newbury, N. R.; Itano, W. M.; Wineland, D. J.; Bergquist, J. C., Frequency ratio of $\mathrm{Al}+$ and $\mathrm{Hg}+$ single-ion optical clocks; metrology at the 17th decimal place, Science $\mathbf{3 1 9}$, 1808, (2008).

[2] S. Schiller, G. M. Tino, P. Gill, C. Salomon, U. Sterr, E. Peik, A. Nevsky, A. Görlitz, D. Svehla, G. Ferrari, N. Poli, L. Lusanna, H. Klein, H. Margolis, P. Lemonde, P. Laurent, G. Santarelli, A. Clairon, W. Ertmer, E. Rasel, J. Mller, L. Iorio, C. Lämmerzahl, H. Dittus, E. Gill, M. Rothacher, F. Flechner, U. Schreiber, V. Flambaum, Wei-Tou Ni, Liang Liu, Xuzong Chen, Jingbiao Chen, Kelin Gao, L. Cacciapuoti, R. Holzwarth, M. P. He and W. Schäfer, Einstein Gravity Explorer - a medium-class fundamental physics mission, Exp. Astron. 23, 573, (2009).

[3] P. Wolf, Ch. J. Bordé, A. Clairon, L. Duchayne, A. Landragin, P. Lemonde, G. Santarelli, W. Ertmer, E. Rasel, F. S. Cataliotti, M. Inguscio, G. M. Tino, P. Gill, H. Klein, S. Reynaud, C. Salomon, E. Peik, O. Bertolami, P. Gil, J. Pramos, C. Jentsch, U. Johann, A. Rathke, P. Bouyer, L. Cacciapuoti, D. Izzo, P. De Natale, B. Christophe, P. Touboul, S. G. Turyshev, J. Anderson, M. E. Tobar, F. Schmidt-Kaler, J. Vigu, A. A. Madej, L. Marmet, M.-C. Angonin, P. Delva, P. Tourrenc, G. Metris, H. Müller, R. Walsworth, Z. H. Lu, L. J. Wang, K. Bongs, A. Toncelli, M. Tonelli, H. Dittus, C. Lämmerzahl, G. Galzerano, P. Laporta, J. Laskar, A. Fienga, F. Roques and K. Sengstock , Quantum physics exploring gravity in the outer solar system: the SAGAS project, Exp. Astron. 23, 651, (2009).

[4] A Bauch, J Achkar, S Bize, D Calonico, R Dach, R Hlavać, L Lorini, T Parker, G Petit, D Piester, K Szymaniec and P Uhrich, Comparison between frequency standards in Europe and the USA at the 10-15 uncertainty level, Metrologia, 43, 109, (2006).

[5] A. Seidel, M.P. Hess, J. Kehrer, W. Schaefer, M. Kufner, M. Siccardi, L. Cacciapuoti, L. Agilar Sanches, S. Feltham. The ACES Microwave Link: Instrument Design And Tests Results, in Proc. of the 21st European Frequency and Time Forum, Geneva, Switzerland, p. 1295, (2007).

[6] Samain Etienne, Guillemot Philippe, Exertier Pierre, Albanese Dominique, Vrancken Patrick, Para Franck, Paris Jocelyn, Torre Jean-Marie, Petitbon Isabelle, Leon Sylvie, Time transfer by laser link - T2L2 : an opportunity to calibrate RF links, in Proc. of the 22nd European Frequency and Time Forum, Toulouse, France, (2008).

[7] Kefelian, F.; Lopez, O.; Jiang, H.; Chardonnet, C.; Amy-Klein, A.; Santarelli, G., Highresolution optical frequency dissemination on a telecommunications network with data traffic, Opt. Lett. 34, 1573, (2009); Grosche, G.; Terra, O.; Predehl, K.; Holzwarth, R.; 
Lipphardt, B.; Vogt, F.; Sterr, U.; Schnatz, H., Optical frequency transfer via $146 \mathrm{~km}$ fiber link with $10^{-19}$ relative accuracy, Opt. Lett. 34, 2270, (2009).

[8] N. Perlot, D. Giggenbach, H. Henniger, J. Horwath, M. Knapek and K. Zettl, Measurements of the Beam-Wave Fluctuations over a 142-km Atmospheric Path, Proc. of SPIE 6304, 63041O, (2006).

[9] A. Alatawi, R. P. Gollapalli, and L. Duan, Radio-frequency clock delivery via free-space frequency comb transmission, Opt. Lett. 34, 3346, (2009).

[10] Sprenger, B.; Zhang, J.; Lu, Z. H.; Wang, L. J., Atmospheric transfer of optical and radio frequency clock signals, Opt. Lett. 34, 965, (2009).

[11] Killinger, D.; Churnside, J.; Rothman, L., Atmospheric Optics, Ch. 44 in Handbook of optics, McGraw-Hill, (1995).

[12] Linfield, R. P.; Colavita, M. M.; Lane, B. F., Atmospheric turbulence measurements with the Palomar testbed interferometer, Astroph. J., 554, 505, (2001). 